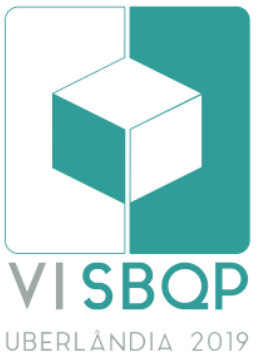

\title{
PROJETAR PARA QUEM? O SER HUMANO NA ARQUITETURA DE MIES VAN DER ROHE
}

\author{
COLOMBO, Luciana Fornari \\ Universidade Federal do Rio Grande do Sul, e-mail: Iuci.colombo@gmail.com
}

\begin{abstract}
RESUMO
Este trabalho de pós-doutorado utiliza a abordagem qualitativa do estudo de caso para investigar como a arquitetura pode atender o ser humano de maneira mais holística, considerando não apenas as suas necessidades corporais, tal como a necessidade de abrigo eficiente e confortável, mas também as suas necessidades espirituais, como o anseio por sentido e significado. O estudo de caso selecionado compreende a filosofia e a arquitetura do renomado arquiteto Ludwig Mies van der Rohe. Este estudo de caso é intrigante, pois, embora enfaticamente explorasse inovações tecnológicas, não pretendia se limitar a tais questões técnicas, materiais e instrumentais. Conforme Mies costumava ressaltar, a arquitetura também deveria atender às necessidades espirituais mais elevadas do ser humano. Por meio da análise de manuscritos, entrevistas, história oral, e biblioteca pessoal do arquiteto, o trabalho esclarece os conceitos de "ser humano", "espírito" e "arquitetura" que sustentavam a filosofia de Mies. A partir deste esclarecimento, o trabalho analisa a arquitetura de Mies, identificando como suas célebres estratégias projetuais se relacionam com o seu objetivo de atender às necessidades espirituais do ser humano. O trabalho não apresenta estas estratégias como soluções prontas a serem imitadas, mas como demonstrações inspiradoras de um direcionamento e de uma postura que reforçam a importância da revitalização de uma visão mais holística do ser humano na arquitetura contemporânea.
\end{abstract}

Palavras-chave: Ludwig Mies van der Rohe, Filosofia da Arquitetura, Arquitetura e Espiritualidade, O Humano na Arquitetura.

\begin{abstract}
This postdoc work uses the case study method as a qualitative research strategy to investigate how architecture can serve human beings more holistically, considering not only their bodily needs, such as the need for efficient, comfortable shelter, but also their spiritual needs, such as the yearning for meaning and significance. The selected case study encompasses the philosophy and the architecture of the renowned architect Ludwig Mies van der Rohe. This case study is intriguing because, though emphatically exploring technological advances, it was not meant to be limited to technical, material, and instrumental questions. As Mies used to highlight, architecture should also strive to serve the highest spiritual needs of the human being. Through the analysis of Mies's manuscripts, interviews, oral history, and personal library, this paper clarifies the concepts of "human being", "spirit", and "architecture" that underpinned his philosophy. In the light of this clarification, the paper analyses Mies's architecture by identifying how his famous design strategies relate to his goal of serving human spiritual needs. The paper does not present these strategies as ready solutions to be imitated, but as inspiring demonstrations of a direction and attitude that reinforce the importance of the revitalization of a more holistic view of the human being in contemporary architecture.
\end{abstract}

Keywords: Ludwig Mies van der Rohe, Philosophy of Architecture, Architecture and Spirituality, The Human in Architecture.

COLOMBO, Luciana Fornari. Projetar para quem? O ser humano na arquitetura de Mies van der Rohe. In: SIMPÓSIO BRASILEIRO DE QUALIDADE DO PROJETO NO AMBIENTE CONSTRUÍDO, 6., 2019, Uberlândia. Anais... Uberlândia: PPGAU/FAUeD/UFU, 2019. p. 502-509. DOI https://doi.org/10.14393/sbqp19047. 


\section{INTRODUÇÃO}

Fotografias de arquitetura frequentemente apresentam espaços sem a presença de pessoas. Tal vazio não significa que o ser humano tenha sido desconsiderado, mas, em geral, apenas que a visualização do edifício foi priorizada. De fato, livros clássicos como A Arte de Projetar em Arquitetura (NEUFERT, 2013) apresentam o ser humano como medida fundamental da arquitetura. Apesar disso, muitas vezes, como no livro citado, o ser humano é considerado apenas como um ente corporal, enquanto outras dimensões humanas, como a religiosa e a espiritual, são omitidas. Essas omissões refletem uma tendência mais ampla à desconsideração do papel da religiosidade e da espiritualidade na cultura moderna ocidental. De acordo com o renomado filósofo Charles Taylor no livro Uma Era Secular (TAYLOR, 2010), esta tendência reflete o entendimento predominante de que a secularização é um processo de esvaziamento de religiosidade e espiritualidade. Questionando este entendimento, Taylor argumenta que a secularização característica da era moderna seria melhor compreendida como um processo de diversificação de posturas religiosos e de experiências de espiritualidade. Em harmonia com este argumento, esforços significativos têm sido feitos para que o papel da religiosidade e da espiritualidade na arquitetura moderna e contemporânea seja mais reconhecido e melhor compreendido. Estes esforços incluem simpósios, como aqueles promovidos pelo The Architecture, Culture, and Spirituality Forum (ACSF, 2007); edições especiais de periódicos, como a edição do European Legacy intitulado A Matter of Interactions-Religion and Architectural Modernism, 1945-70 (HEYNICKX; SYMONS, 2017); e livros, como The Religious Imagination in Modern and Contemporary Architecture: A Reader (HEJDUK; WILLIAMSON, 2011). Contribuindo com estes esforços, o presente trabalho pretende investigar maneiras pelas quais a arquitetura pode atender o ser humano de maneira mais holística, considerando não apenas as necessidades corporais, como a necessidade de abrigo confortável e eficiente, mas também as suas necessidades espirituais, como o anseio por propósito e significado.

\section{METODOLOGIA}

Para alcançar este objetivo o trabalho adota a estratégia de pesquisa qualitativa conhecida como estudo de caso. O estudo de caso selecionado compreende a filosofia e a arquitetura do renomado arquiteto Ludwig Mies van der Rohe (Aachen, 1886-Chicago, 1969). Este caso é intrigante, pois, embora enfaticamente explorasse inovações tecnológicas, não pretendia se limitar a tais questões técnicas, materiais e instrumentais. Conforme Mies costumava ressaltar, a arquitetura também deveria atender às necessidades espirituais mais elevadas do ser humano. Este posicionamento é apresentado, por exemplo, na seguinte definição de arquitetura apresentada por ele em 1928:

A arquitetura [Baukunst] consiste no diálogo espacial entre o ser humano e o seu meio ambiente, demonstrando como o ser humano se impõem neste ambiente e o domina. Por esta razão, a arquitetura não é meramente um problema técnico, nem um problema de organização e economia. Na realidade, a arquitetura sempre é a manifestação espacial de decisões espirituais (MIES VAN DER ROHE, 1991a, p. 299). 
O estudo de caso selecionado também é relevante, pois, embora já tenha sido estudado do ponto de vista do papel da espiritualidade na arquitetura (ex.: MARTIGNAGO, 2015; MERTINS, 2014a; NEUMEYER, 1991; PADOVAN, 2002), este caso ainda pode ser melhor compreendido. Conforme apontado por Detlef Mertins em Mies (2014b, p. 155), ainda seria preciso considerar o conceito Miesiano de espírito não apenas do ponto de vista artístico e filosófico, mas também teológico, visto que os referenciais teóricos do arquiteto demonstram uma preocupação explícita com questões teológicas.

Considerando esta necessidade de aprofundamento, o presente trabalho investiga o discurso de Mies disponível em publicações e arquivos buscando esclarecer o que este arquiteto entendia por "espírito". O trabalho examina como este conceito se relaciona com os conceitos Miesianos de "ser humano" e "arquitetura" e com os referenciais teóricos de Mies, dentre os quais se destaca a obra do filósofo e teólogo Tomás de Aquino. Por fim, o trabalho examina como estes conceitos se relacionam com as estratégias projetuais desenvolvidas por Mies tendo em vista o objetivo de atender à dimensão espiritual do ser humano.

\section{RESULTADOS E DISCUSSÃO}

\subsection{Conceitos}

Seguindo o pensamento de Tomás de Aquino, Mies entedia o "ser humano" como um ser racional, dotado de livre arbítrio e composto de corpo e espírito. Mies costumava citar a seguinte frase de Tomás de Aquino, "a razão é o princípio fundamental de toda obra humana" (AQUINAS, 2017 I-II, q.90; MIES VAN DER ROHE, 2008, p. 56). Tomás de Aquino entendia a razão como "a transição de uma coisa para outra a partir da qual a alma humana alcança conhecimento sobre algo" (AQUINAS, 1952 q.15). Este filósofo associava a racionalidade com outras características fundamentais do ser humano: o livrearbítrio, quer dizer, "o poder de escolha" (AQUINAS, 2017 I, q.83) e o espírito-O poder que, em última instância, impele o ser humano a agir e conhecer (AQUINAS, [s.d.]).

Em conformidade com esta visão do ser humano, Mies desenvolveu um processo projetual que envolvia uma séria de decisões baseadas em critérios bem fundamentados e no teste de inúmeras alternativas, do partido aos detalhes. Mies ressaltou a importância deste processo exaustivo, "você pode cometer erros, mas jamais deve construí-IOs" (MIES VAN DER ROHE, 1958). Além de reconhecer a racionalidade humana por meio de soluções coerentes, consistentes e objetivas, a arquitetura de Mies também buscava reconhecer o livre arbítrio do ser humano ao oferecer espaços flexíveis: "projete o edifício amplo o bastante para que se possa caminhar nele de maneira livre e não apenas em uma direção predeterminada. Não fazemos ideia se as pessoas usarão o edifício da maneira como imaginamos. Primeiramente, os usos não são claros e, segundo, não são constantes-eles mudam muito mais rápido que o edifício" (MIES VAN DER ROHE, 1966, p. 4). Também reconhecendo a dimensão espiritual do ser humano, Mies ressaltou que as decisões tomadas durante o processo de projeto deveriam atender às necessidades não apenas físicas, como a necessidade de abrigo, mas também espirituais, como o anseio 
por significado e propósito, o qual distingue o ser humano de outros animais (MIES VAN DER ROHE, 1991b, p. 274).

Mies compreendeu que estes anseios espirituais vinham sendo reprimidos na era moderna devido a uma valorização excessiva da tecnologia. O rápido desenvolvimento tecnológico chegava a níveis tão impressionantes que tendia a ser visto com um fim em si mesmo. Entretanto, Mies alertava, a tecnologia continuava sendo um meio, um instrumento. A arquitetura, mesmo na era tecnológica, só faria sentido se utilizasse estes meios para promover o progresso espiritual do ser humano e atingir fins espirituais mais elevados, como a verdade, a bondade e a beleza (MIES VAN DER ROHE, 1991c, p. 250, 1991d, p. 3111 .

Seguindo o realismo moderado de Tomás de Aquino, Mies acreditava que todas as coisas eram naturalmente verdadeiras, boas e belas, ainda que em graus diferentes. Quanto mais perfeitamente uma coisa manifestava a sua essência, natureza e ordem, mais intensamente estas propriedades estariam presentes. Para intensificar a presença destas propriedades na arquitetura, Mies se valia do princípio da clareza, o qual consistia na realização e expressão de essências da maneira mais natural, lógica e inteligível possível. A busca por clareza levaria Mies a desenvolver uma arquitetura sutil e serena, focada no domínio e refinamento de elementos intrínsecos à arquitetura. Quando vista como fundo ou moldura, esta arquitetura realçava a essência, o propósito e a beleza dos elementos circundantes, como vistas, vegetação, pessoas, objetos e ações do cotidiano. Quando vista como figura, ela realçava a essência, o propósito e a beleza dos elementos construtivos ao expô-los de maneira coerente e inteligível e ao refiná-los considerando as suas proporções, disposição, efeitos de luz e sombra, detalhamento e simbolismo (Figura 1).

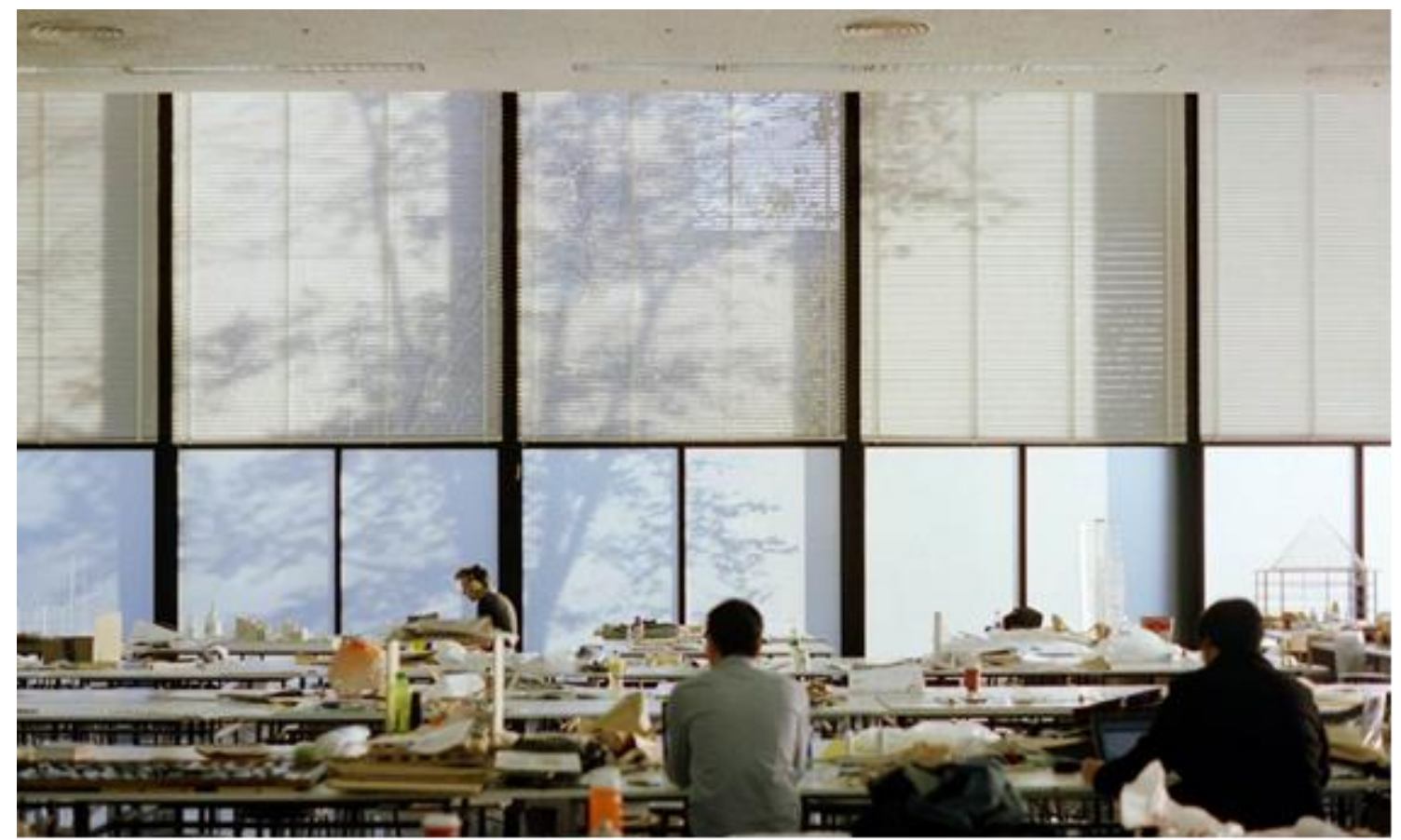

Figura 1 - S.R. Crown Hall, Chicago, 1956, Arquiteto Mies van der Rohe.

Fonte: Timothy Brown (2014, CC BY 2.0, via Flickr) 


\subsection{Estratégias}

A marcante pele de vidro desenvolvida por Mies em sua arquitetura contribuiu estrategicamente para a sua busca por clareza. Substituindo a pesada e opaca parede de alvenaria tradicional, esta fina e transparente pele de vidro promovia clareza não apenas no sentido físico de reflexão e transmissão de luz, mas também no sentido de entendimento e legibilidade, já que sua aparência imaterial demonstrava o princípio construtivo moderno de concentração das cargas no esqueleto. Além disso, a pele de vidro aproveitava melhor a independência das paredes em relação ao sistema de sustentação do edifício para otimizar vistas e integração espacial.

Mies começou a explorar estas potencialidades da pele de vidro no seu projeto de edifício alto localizado em um terreno triangular em Berlim, o qual desenvolveu para um concurso no início da década de 1920. Este projeto tinha forte caráter experimental, visto que o contexto pós-guerra de hiperinflação e instabilidade reduzia dramaticamente as oportunidades de construção. Durante os seus experimentos com a pele de vidro, Mies buscou realçar os reflexos luminosos, enquanto evitava o ofuscamento e o efeito especular excessivos. Para tanto, ele angulou as amplas superfícies de vidro até que estas adquirissem o caráter resplandecente de um cristal. Durante o dia, a pele de vidro refletia a luz natural externa, e, durante a noite, transmitia a luz artificial interna, se tornando transparente e expondo o esqueleto do edifício (MIES VAN DER ROHE, 1951, 1971, p. 34, 1991e, p. 240).

Além da pele de vidro, os protuberantes perfis metálicos desenvolvidos por Mies na fase americana de sua carreira também contribuíram estrategicamente para a sua busca por clareza. Em projetos como a Casa Farnsworth (1946-51) e a Crown Hall (1950-56), estes perfis eram pilares que, exteriorizados, tornavam o esqueleto do edifício mais evidente e inteligível. Já em projetos como o IIT Alumni Memorial Hall (1945-46) e as torres de apartamentos no 860-880 Lake Shore Drive (1948-51), estes perfis, embora já não fizessem parte do esqueleto, ainda evocavam a sua presença por similaridade (PADOVAN, 2002, p. 166-168; SCHULZE; WINDHORST, 2012, p. 289). Nestes casos, o esqueleto não pôde ser exposto diretamente devido ao revestimento de concreto exigido pela legislação de prevenção a incêndios em prédios. Além de tornar a materialidade do edifício mais inteligível, estes perfis também contribuíam para refinar a aparência do edifício em termos de ritmo, unidade, e efeitos de luz e sombra; e para resolver problemas construtivos, como a fixação do vidro, o encontro das divisórias internas com a fachada e a resistência a cargas laterais (MIES VAN DER ROHE, 1950s, p. 11, 1952, p. 99, 1958, p. 9).

A busca de Mies pela expressão de materiais e técnicas construtivas tinha como objetivo esclarecer não apenas o sistema estrutural do edifício, mas também as circunstâncias históricas que haviam influenciado e possibilitado a sua realização. Por exemplo, Mies preferia utilizar o aço ao concreto armado, porque, além de ter um processo de montagem mais rápido e eficiente, o aço também tinha uma aparência mais leve, esbelta e precisa que era mais representativa da modernidade do que a aparência robusta, rugosa e pesada do concreto armado (MIES VAN DER ROHE, 2008, p. 72). De fato, o concreto era um material utilizado desde a antiguidade e que havia sido modernizado, possibilitando maiores vãos e alturas, precisamente através da introdução de reforços de aço em seu interior. Esta busca mais ampla pela 
expressão de circunstâncias históricas ajuda a explicar porque Mies utilizou revestimento metálico em alguns projetos construídos com esqueleto de concreto armado, como as torres de apartamentos no 900-910 Lake Shore Drive (1953-56), embora já tivesse desenvolvido um vocabulário arquitetônico próprio para expressar a plasticidade do concreto, como exemplificado no edifício Promontory (1946-49). No caso das torres no 900-910 Lake Shore Drive, essa decisão projetual singular seria reforçada pela busca por homogeneidade e continuidade visual com as torres vizinhas construídas no 860-880 Lake Shore Drive em esqueleto de aço.

\section{CONCLUSÃO}

O presente trabalho analisou a filosofia e a arquitetura de Mies van der Rohe a fim de identificar maneiras por meio das quais este arquiteto buscou atender às aspirações espirituais do ser humano. Aceitando o anseio moderno por desenvolvimento tecnológico, mas rejeitando este desenvolvimento como um fim em si mesmo, Mies buscava uma arquitetura que colocasse a tecnologia a serviço do ser humano e de sua distintiva aspiração espiritual por sentido e significado. Seguindo a filosofia de Tomás de Aquino, Mies se apoiava no princípio da clareza para desenvolver uma arquitetura sutil e serena capaz de realçar a essência, o propósito e o significado de suas partes e de elementos circundantes; e de intensificar a verdade, bondade e beleza intrínsecas às coisas. Mies se valia de elementos intrínsecos à arquitetura, como pele de vidro e perfis metálicos, para refinar o edifício e demonstrar os princípios construtivos e as circunstâncias históricas que caracterizavam e viabilizavam 0 desenvolvimento da arquitetura moderna. Mais do que ilustrar soluções prontas a serem imitadas, estas célebres estratégias projetuais utilizadas por Mies demonstram um direcionamento e uma postura capazes de promover um atendimento mais holística e profundo das necessidades humanas na arquitetura contemporânea.

\section{REFERÊNCIAS}

ACSF. The Architecture, Culture, and Spirituality Forum, 2007. Disponível em: $<$ http://acsforum.org>

AQUINAS, T. The Disputed Questions on Truth. Tradução Robert W. Mulligan; James V. McGlynn; Robert W. Schmidt. Chicago: Henry Regnery Company, 1952.

AQUINAS, T. The Summa Theologica of St. Thomas Aquinas. Tradução Fathers of the English Dominican Province. London: Burns Oates and Washbourne, 2017.

AQUINAS, T. Commentary On the First Epistle to the Corinthians. Tradução Fabian Larcher. [s.l: s.n.].

HEJDUK, R.; WILLIAMSON, J. (EDS.). The Religious Imagination in Modern and Contemporary Architecture: A Reader. New York: Routledge, 2011.

HEYNICKX, R.; SYMONS, S. A Matter of Interactions-Religion and Architectural Modernism, 1945-70: Introduction. The European Legacy, v. 22, n. 3, p. 251-257, 3 abr. 2017.

KUCKO, J. An Exploratory Journey of Spirituality in Design and Architecture. Journal of Interior Design, v. 44, n. 1, p. 3-4, 2019. 
MARTIGNAGO, M. DAL C. Mies van der Rohe e as Decisões do Espírito: Leituras Sobre a Imaterialidade na Obra do Pavilhão de Barcelona. Dissertação de Mestrado-São Paulo: Universidade Presbiteriana Mackenzie, 2015.

MERTINS, D. Barcelona Pavilion: Spiritualizing Technology. In: Mies. London; New York: Phaidon Press, 2014a. p. 138-167.

MERTINS, D. Mies. London; New York: Phaidon Press, $2014 \mathrm{~b}$.

MIES VAN DER ROHE, L. Informal discussion with students and young architects at the Architectural League NY, 1950s. Papers of Ludwig Mies van der Rohe, Library of Congress, Washington DC.

MIES VAN DER ROHE, L. Letter to Mr. Don J Burg. Papers of Ludwig Mies van der Rohe, 1951. Papers of Ludwig Mies van der Rohe, Library of Congress, Washington DC.

MIES VAN DER ROHE, L. Mies van der Rohe. Architectural Forum, p. 93-110, nov. 1952.

MIES VAN DER ROHE, L. Script Seagram film, excerpts from interviews, 12 fev. 1958. Papers of Ludwig Mies van der Rohe, Library of Congress, Washington DC.

MIES VAN DER ROHE, L. Mies in Berlin, interview by Ulrich Conrads, transcript, 1966. Papers of Ludwig Mies van der Rohe, Library of Congress, Washington DC.

MIES VAN DER ROHE, L. Interview by Katherine Kuh (1964). In: KUH, K. (Ed.). The open eye: in pursuit of art. New York: Harper \& Row, 1971.

MIES VAN DER ROHE, L. The Preconditions of Architectural Work (1928). In: NEUMEYER, F. (Ed.). The Artless Word: Mies van der Rohe on the Building Art. Cambridge, Mass.: MIT Press, 1991a. p. 299-301.

MIES VAN DER ROHE, L. Notebook (1927-8). In: NEUMEYER, F. (Ed.). The Artless Word: Mies van der Rohe on the Building Art. Cambridge, Mass.: MIT Press, 1991b. p. 265-296.

MIES VAN DER ROHE, L. Lecture (19 June 1924). In: NEUMEYER, F. (Ed.). The Artless Word: Mies van der Rohe on the Building Art. Cambridge, Mass.: MIT Press, 1991c. p. 249-250.

MIES VAN DER ROHE, L. Speech on the Occasion of the Anniversary Meeting of the Deutscher Werkbund (1932). In: NEUMEYER, F. (Ed.). The Artless Word: Mies van der Rohe on the Building Art. Cambridge, Mass.: MIT Press, 1991d. p. 311.

MIES VAN DER ROHE, L. Skyscrapers, 1922. In: NEUMEYER, F. (Ed.). The Artless Word: Mies van der Rohe on the Building Art. Cambridge, Mass.: MIT Press, 1991 le. p. 240-240.

MIES VAN DER ROHE, L. Conversation Three (New York, 1955; Chicago, 1964). In: PUENTE, M. (Ed.). Conversations with Mies van der Rohe. New York: Princeton Architectural, 2008. p. 49-88.

NEUFERT, E. Arte de Projetar em Arquitetura. 18. ed. Barcelona: Gustavo Gili, 2013.

NEUMEYER, F. From Material through Purpose to ldea: The Long Path to the Building Art. In: The Artless Word: Mies van der Rohe on the Building Art.

Tradução Mark Jarzombek. Cambridge, Mass.: MIT Press, 1991. p. 145-194. 
PADOVAN, R. Mies: The Correspondence of Thing and Intellect. In: Towards

Universality: Le Corbusier, Mies and De Stijl. London; New York: Routledge, 2002. p. 146-173.

SCHULZE, F.; WINDHORST, E. Mies van der Rohe: A Critical Biography, New and Revised Edition. Chicago: University of Chicago Press, 2012.

TAYLOR, C. Uma Era Secular. São Leopoldo: Unisinos, 2010. 